\title{
Dinâmica do peso e da condição corporal e eficiência reprodutiva de ovelhas da raça Santa Inês e mestiças Santa Inês-Suffolk submetidas a dois sistemas de alimentação em intervalos entre partos de oito meses ${ }^{1}$
}

\author{
Dynamic of weight and corporal score and reproductive efficiency of the Santa Ines breed and crossbreed \\ Suffolk ewes submitted to two feeding systems and lambing of eight months
}

\author{
Claudia da Costa Boucinhas ${ }^{2}$ Edson Ramos de Siqueira ${ }^{3}$ Sirlei Aparecida Maestá ${ }^{4}$
}

\section{RESUMO}

O trabalho teve como objetivo comparar a eficiencia produtiva e reprodutiva de ovelhas em dois sistemas de alimentação. Noventa e oito matrizes da raça Santa Inês e trinta e nove mestiças Suffolk (3/4 Suffolk + 1/4 Santa Inês) foram divididas em dois tratamentos: tratamento 1 (T1) mantidas em pastagem, com suplementação de silagem de capim elefante na seca invernal, e tratamento 2 (T2) -mantidas em pastagem, com suplementação de silagem de capim elefante na seca invernal e de concentrado três semanas antes e durante a estação de monta, três semanas antes do parto e durante a lactação. Verificou-se diferença $(P<0,05)$ entre peso inicial (PI) $(52,5 \mathrm{~kg}$ e $54,33 \mathrm{~kg})$, e peso final (PF) $(53,38 \mathrm{~kg}$ e $55,76 \mathrm{~kg})$ para $T 1$ e T2, respectivamente. Houve efeito de genótipo $(P<0,05)$, sendo PI 51,46kg para a raça Santa Inês (SI) e $55,38 \mathrm{~kg}$ para as mestiças Santa Inês-Suffolk (SF). Para PF, observou-se 52,36kg para a Santa Inês e 56,78kg na mestiça Santa Inês-Suffolk. O peso pré-parto $(P P P)$ diferiu $(P<0,05)$ apenas entre as estações, tendo sido de $65,23 \mathrm{~kg}$ na estação reprodutiva I (2 a 4/2002), 58,15kg na estação reprodutiva II (10 a 12/2002) e 59,73kg na estação reprodutiva III (6 a 8/ 2003). No peso pós-parto (PPART), também ocorreram diferenças $(P<0,05)$ entre a raça Santa Inês $(53,59 \mathrm{~kg})$ e a mestiça Santa Inês-Suffolk (57,05kg); no peso aos 30 dias de lactação (P30d) a Santa Inês registrou 52,94kg e a mestiça Santa Inês-Suffolk 55,45kg. O peso aos 70 dias (P70d) de lactação foi para a Santa Inês de 50,83kg e de 53,22 kg para a mestiça Santa Inês-Suffolk; e o peso aos 100dias (P100d) de lactação foi de $51,55 \mathrm{~kg}$ e de $53,61 \mathrm{~kg}$ para a Santa Inês e para a mestiça Santa Inês-Suffolk, respectivamente. A condição corporal inicial $(C C I)$ foi maior $(P<0,05)$ para o T2 2,47 do que para o T1 2,16. Na condição corporal final (CCF), 2,19e 2,6, respectivamente para $T 1$ e T2, mas os tratamentos não diferiram na condição corporal pré-parto (CCPP). A CCI 2,4 para a mestiça Santa Inês-Suffolk foi $(P<0,05)$ em relação a Santa Inês 2,22. A CCF da mestiça Santa Inês-Suffolk de 2,49 também foi maior $(P<0,05)$ que da Santa Inês que obteve 2,3, mas não diferiram na CCPP. Em relação as três estações reprodutivas, apenas na estação I a CCI 2,55, CCF 2,8 e a CСPP 3,03 foram maiores $(P<0,05)$. Já as estações reprodutivas $I I=2,47$ e $I I I=2,1$ diferiram somente na CCPP que foi menor na estação III. Na estação I e T1 ambos os genótipos obtiveram $72,5 \%$ de fertilidade e o T2 apresentou para as mestiças Santa Inês-Suffolk $77 \%$ e para a Santa Inês 88\%. Na estação II o T1 obteve para as mestiças Santa InêsSuffolk 42\% e para as Santa Inês 38\% e o T2 resultou em 56 e $50 \%$ para as mestiças Santa Inês-Suffolk e Santa Inês respectivamente. Na estação III a fertilidade do T1 foi para as mestiças $60 \%$ e para as Santa Inês $54 \%$ e o T2 $87 \%$ e $76 \%$ para as mestiças e Santa Inês respectivamente. Encontrou-se diferença na prolificidade, entre os tratamentos, sendo na estação I a prolificidade foi de 1,20 e 1,55, na estação II foi 0,90 e 1,03 e na estação III obteve-se 1,11 e 1,14, respectivamente para o $T 1$ e T2. Concluiu-se que a suplementação melhorou o desempenho reprodutivo das ovelhas.

Palavras-chave: ovinos, ovelha condição corporal, eficiência reprodutiva.

\section{ABSTRACT}

This study was carried out with the objective of comparing the productive and reproductive efficiency of ewes in two productive systems. Ninety eight Santa Ines ewes and thirty nine crossbred Suffolk were divide in two treatments: T1maintained on pasture, with supplementation of Pennisetum purpureum silage in dry winter, T2- maintained on pasture, with supplementation of Pennisetum purpureum silage in winter

${ }^{1}$ Programa de Pós-graduação em Zootecnia, Universidade Estadual Paulista (UNESP), Faculdade de Medicina Veterinária e Zootecnia (FMVZ), Caixa Postal 560, 18618-000, Botucatu, SP, Brasil. Email: clabou@laser.com.br. Autor para correspondência.

${ }^{2}$ Departamento de Produção e Exploração Animal da FMVZ, Campus de Botucatu, SP, Brasil.

${ }^{3}$ Pós-Doutorado, UNESP, FMVZ, Botucatu, SP, Brasil. 
and grain ration for three weeks before and during the mating, three weeks before lambing and during the lactation. There was difference $(P<0.05)$ between initial weight $(I W) ;(52.50$ and $54.33 \mathrm{~kg})$ and final weight $(F W) ;(53.53$ and $55.76 \mathrm{~kg})$ for $T 1$ and $T 2$ respectively; and breed effect $(P<0.05)$ : the IW was 51.46 for SI and 55.38 to crossbreed SF, and the FW was 52.36 for SI and $56.78 \mathrm{~kg}$ for crossbreed SF. The pre-lambing weight (PL) was different only among the seasons $(65.23 \mathrm{~kg}$ for Season I, 58.15kg for Se II and 59.73kg to Se III). The after lambing weight $(A L)$ was also different $(P<0.05)$ between breeds ( $S I$ $53.59 \mathrm{~kg}$ and crossbreed SF $57.05 \mathrm{~kg}$ ), and so the weight 30 days after (W30d) when SI presented $52.94 \mathrm{~kg}$ and crossbreed SF $55.45 \mathrm{~kg}$. The ewe weights 70 days (W70d) after birth were for SI 50.83 and 53.22 for crossbreed SF and the weights in the $100^{\text {th }}$ day (W100d) were 51.55 and $53.61 \mathrm{~kg}$ for SI and crossbreed SF, respectively. The initial corporal score (ICS) was higher for $T 2$ (2.47) when compared to $T 1$ (2.16). The final corporal scores (FCS) were 2.19 and 2.60, respectively to $T 1$ and T2 $(P<0.05)$, but there was not difference between treatments in the pre- lambing corporal score (PCS). The ICS of 2.40 for crossbreed SF was $(P<0.05)$ higher than for $S I$ (2.22). In the three reproductive seasons, only in Se I the SCI (2.55), SCF (2.80) and SCB (3.03) were significantly higher. However, the PCS was slower in Se III (2.10) than in II (2.47). The ewes in Se I and T1 had $72.5 \%$ of fertility and in the T2 they had 82.5\%; in Se II the females from $\mathrm{T} 1$ had $40.0 \%$ and those from T2 had 53.0\%. The fertility in the Se III was $57.0 \%$ for $T 1$ and had $81.5 \%$ for T2. The prolificity in the Se I was 1.20 and 1.55 for T1 and T2 respectively; in the Se II 0.90 and 1.03 and for Se III, 1.11 and 1.14. It was concluded that the supplementation had increased the productive performance of ewes.

Key words: ovine, sheep, score corporal, efficiency reproductive.

\section{INTRODUÇÃO}

No Estado de São Paulo e em estados vizinhos, a produção de carne ovina baseia-se em raças exóticas, caracterizadas por apresentarem estacionalidade reprodutiva. Este detalhe pode dificultar a disponibilização de carne ao longo de todo ano, conforme exige o mercado atual. As raças sem anestro sazonal facilitam a diminuição do intervalo de partos, podendo-se ter como objetivo a obtenção de três partos a cada dois anos.

No contexto técnico-econômico, a alimentação é ponto chave, pouco sabendo-se a respeito da relação custo-benefício. Sendo assim, urge a realização de estudos que envolvam o estabelecimento não apenas de sistemas tecnicamente viáveis, mas de modelos eficazes sob a ótica empresarial.

O peso corporal em ovinos é visto como uma medida indireta e pouco eficaz para se avaliar o estado nutricional, devido às diferentes raças com presença ou não de lã, aos tipos de gestações e ao estado do animal. Com isso, vários trabalhos têm utilizado a avaliação da condição corporal (CC) como um método eficaz e simples para se obter o estado nutricional do rebanho (DUCKER \& BOYD, 1977; GUNN etal. 1984). DUCKER \& BOYD (1977) observaram que ovelhas de pequeno porte e alta CC tiveram maior taxa de ovulação do que ovelhas maiores com baixa CC. Mas GUNN et al. (1984) constataram pouca influência do estado nutricional na taxa de ovulação, no decorrer do período de monta, quando a CC média das ovelhas foi de 2,5, sendo este sugerido como escore corporal médio para se obter taxas de ovulação satisfatórias. RIBEIRO et al. (2003) afirmam que as perdas reprodutivas que ocorrem em rebanhos ovinos no RS devido a infertilidade puderam ser relacionados com CC no encarneiramento.

Este estudo teve como objetivo avaliar a eficiência produtiva e reprodutiva das ovelhas da raça Santa Inês e de mestiças Suffolk em regime de três partos a cada dois anos, mantidas em pastagem, suplementadas ou não com concentrados.

\section{MATERIAL E MÉTODOS}

O experimento foi conduzido na Fazenda São José, localizada no município de Bofete-SP. Foram utilizadas 98 fêmeas da raça Santa Inês (SI) e 39 mestiças Suffolk ( $1 / 4$ Santa Inês+3/4 Suffolk) (SIxSF), subdivididas aleatoriamente em dois tratamentos, onde as ovelhas permaneceram no mesmo tratamento durante todo o experimento.

O tratamento 1 (não suplementado) e o tratamento 2 (suplementado) foram devidamente balanceados para genótipo e idade (primíparas, ovelhas de segunda cria, ovelhas com mais de duas crias, com idade máxima de cinco anos). Os lotes foram mantidos em pastagem de Brachiária humidícula e Brachiaria decumbens e Panicum maximum cv. Tanzânia, com fornecimento de silagem de capim elefante (Pennisetum purpureum) na seca invernal, quando a má condição do pasto indicou necessidade. As ovelhas do tratamento 2 foram suplementadas com concentrado, de acordo com o (NRC, 1985), com 90,45 \% de (MS), $75 \%$ de (NDT), $13 \%$ de (PB) e $9,34 \%$ de (FB), composto por: rolão de milho, polpa cítrica e farelo de trigo, na quantia de $500 \mathrm{~g}$ por cabeça por dia, três semanas antes e quatro após o início do manejo para concepção, três semanas antes do parto e durante os 70 dias ou 100 dias de lactação. Ambos os tratamentos dispuseram de mistura mineral à vontade. Utilizou-se o manejo rotacionado de pastagem (oito piquetes de 5 hectares cada) a cada 15 dias, de forma que os dois lotes pastejaram toda a área de maneira homogênea. Amostras das pastagens foram coletadas mensalmente para as análises bromatológicas. 
As ovelhas foram submetidas ao efeito macho, sendo os reprodutores isolados, durante trinta dias, das fêmeas. O manejo reprodutivo visou à obtenção de três partos em dois anos. Para evitar o efeito de reprodutor, foi feito rodízio, possibilitando que, de acordo com as raças, os carneiros atendessem aos dois lotes alternadamente. Os carneiros foram soltos diretamente com as ovelhas, durante sessenta dias; os acasalamentos foram em fev/abril de 2002 (Est.I), out/dez de 2002 (Est.II) e jun/ag de 2003 (Est.III).

De acordo com as médias de OPG (ovos por grama de fezes), acima de 500, obtidas pelo exame de fezes através da técnica de Gordon e Whitlock (1939), as ovelhas foram tratadas com anti-helmíntico (Cidectin, Fort Dodge).

Considerou-se as seguintes variáveis:

- peso e condição corporal (escala de 1 a 5 , fracionada em 0,25 ) das ovelhas, nos seguintes momentos: ao início da estação de monta, 30 dias após o início da referida estação, três semanas antes do parto, ao parto, 30, 70 e 100 dias após o parto.

- fertilidade das ovelhas (número de ovelhas paridas $\div$ número de ovelhas colocadas em reprodução) $\mathrm{x} 100$;

- prolificidade (número de cordeiros nascidos $\div$ número de ovelhas paridas) x 100 .

As análises estatísticas foram processadas no Sistema para Análises Estatísticas e Genéticas SAEG (UFV, 2000). O delineamento foi inteiramente casualizado, levando em consideração os efeitos de tratamento, raça, época e suas interações (trat*raça, trat*época, raça*época). Para os dados de peso inicial e final, ao parir, 30, 70 e 100 dias pós-parto das ovelhas, utilizou-se a análise de variância. As médias foram comparadas pelo teste de Tukey, com probabilidade de $5 \%$. Para os dados não paramétricos (escore corporal), utilizou-se o Teste de Wilcoxon para os dados não paramétricos, para as características com duas variáveis [raça Santa Inês (SI) ou mestiça Santa InêsSuffolk (SIxSF) e tratamento 1 (suplementado) e 2 (não suplementado)]; e o Teste de Kruskal-Wallis, quando apresentavam três variáveis (estação reprodutiva I, II e III).

\section{RESULTADOS E DISCUSSÃO}

Na tabela 1, são apresentadas as médias para peso inicial (PI), peso final (PF) e peso pré-parto (PPP) durante as estações reprodutivas I, II e III. Em relação aos tratamentos, verificou-se diferença significativa $(\mathrm{P}<0,05)$ entre PI $(52,5 \mathrm{~kg}$ e $54,33 \mathrm{~kg})$ e PF (53,38kg e 55,76kg) para T1 e T2, respectivamente, demonstrando que o melhor aporte nutricional proporcionou maior peso. MORLEY et al. (1978) constataram que o peso corporal influencia a taxa de ovulação das ovelhas, tendo obtido, em rebanho da raça Merino, 2-2,5\% de aumento da taxa de ovulação para cada quilo de aumento de peso corporal.

Ocorreram diferenças $(\mathrm{P}<0,05)$, como era de se esperar, entre os genótipos devido ao maior porte

Tabela 1 - Médias de peso inicial (PI), peso final(PF) da estação reprodutiva, peso pré-parto(PPP), peso ao parto (PPART), peso aos 30 dias (P30d), 70 dias (P70d) e 100 dias (P100d) pós-parto, da raça SI e mestiças SIxSF, nos tratamentos 1 e 2, durante as estações reprodutivas I, II e III.

\begin{tabular}{|c|c|c|c|c|c|c|c|c|c|}
\hline & $\mathrm{T} 1$ & $\mathrm{~T} 2$ & SI & SF & EST.I & EST.II & EST.III & C.V.(\%) & $\begin{array}{l}\text { Desvio } \\
\text { Padrão }\end{array}$ \\
\hline PI & $52,51^{\mathrm{a}}$ & $54,33^{b}$ & $51,46^{\mathrm{a}}$ & $55,38^{\mathrm{b}}$ & 53,98 & 52,35 & 53,92 & 13,31 & 7,3533 \\
\hline $\mathrm{PF}$ & $53,38^{\mathrm{a}}$ & $55,76^{\mathrm{b}}$ & $52,36^{\mathrm{a}}$ & $56,78^{\mathrm{b}}$ & 54,84 & 53,49 & 55,38 & 13,19 & 7,5315 \\
\hline PPP & 59,57 & 62,50 & 58,92 & 63,16 & $65,23^{b}$ & $58,15^{\mathrm{a}}$ & $59,73^{\mathrm{a}}$ & 12,50 & 8,4741 \\
\hline PPARTO & $54,44^{\mathrm{b}}$ & $56,47^{\mathrm{a}}$ & $53,59^{b}$ & $57,32^{\mathrm{a}}$ & 57,05 & 54,43 & 54,88 & 12,73 & 7,3140 \\
\hline P30d & $52,92^{\mathrm{b}}$ & $55,47^{\mathrm{a}}$ & $52,94^{\mathrm{b}}$ & $55,45^{\mathrm{a}}$ & 53,36 & 55,76 & 53,46 & 12,46 & 6,9477 \\
\hline P70d & $50,64^{\mathrm{b}}$ & $53,41^{\mathrm{a}}$ & $50,83^{\mathrm{b}}$ & $53,22^{\mathrm{a}}$ & 50,88 & 52,99 & 52,20 & 11,95 & 6,4962 \\
\hline P100d & $50,54^{\mathrm{b}}$ & $54,62^{\mathrm{a}}$ & $51,55^{\mathrm{b}}$ & $53,61^{\mathrm{a}}$ & $51,34^{\mathrm{b}}$ & $52,10^{\mathrm{ab}}$ & $54,30^{\mathrm{a}}$ & 12,21 & 6,9100 \\
\hline
\end{tabular}

Médias seguidas de letras distintas na mesma linha diferem entre si pelo Teste de Tukey. T1: não suplementadas,T2:suplementadas.

Ciência Rural, v.36, n.3, mai-jun, 2006. 
das ovelhas mestiças, sendo PI= 51,46kg para a Santa Inês e de 55,38kg para a mestiça Santa Inês-Suffolk. Para o PF, observou-se 52,36kg para a Santa Inês e $56,78 \mathrm{~kg}$ na mestiça Santa Inês-Suffolk. O peso pré parto (PPP) diferiu $(\mathrm{P}<0,05)$ apenas entre as estações reprodutivas, tendo sido de $65,23 \mathrm{~kg}$ na estação I, 58,15kg na estação II e 59,73kg na estação III. Esse maior peso obtido na primeira estação reprodutiva provavelmente ocorreu pelo fato de as ovelhas estarem menos desgastadas no início do sistema de parição, com intervalo entre partos de 8 meses.

Na tabela 1, estão também os resultados para peso: ao parto, aos 30 dias, 70 dias e 100 dias de lactação. Os tratamentos diferiram $(\mathrm{P}<0,05)$ no peso ao parto $(54,44 \mathrm{~kg}$ e $56,47 \mathrm{~kg})$, aos 30 dias de lactação (52,92kg e 55,47kg), aos 70 dias (50,64kg e 53,41kg), e aos 100 dias (50,54kg e 54,62kg), respectivamente para os T1 e T2. Segundo SUSIN (1996), a escala da perda de peso pode variar, em virtude da a qualidade e quantidade do alimento disponível, do número de cordeiros amamentados, de fatores ambientais e do potencial produtivo da matriz. Também houve efeito de genótipo $(\mathrm{P}<0,05)$ no peso ao parto, sendo a média de 53,59kg para a Santa Inês e de 57,05kg para a mestiça Santa Inês-Suffolk, a média no peso aos 30dias para a Santa Inês foi de 52,94kg e de 55,45kg para a mestiça Santa Inês-Suffolk. Aos setenta dias de lactação, a média de peso foi de 50,83kg e 53,22kg, e, aos 100 dias de lactação, a média do peso foi 51,55kg e 53,61kg para a Santa Inês e mestiça santa Inês-Suffolk, respectivamente. O maior peso para as ovelhas mestiças Santa Inês-Suffolk já era esperado, devido ao seu maior porte em relação à Santa Inês. Quanto ao efeito de época, somente na estação III o peso das ovelhas aos
100 dias de lactação $(54,30 \mathrm{~kg})$ foi maior, comparado com os das estações I (51,34kg) e II (52,10kg).

$\mathrm{Na}$ estação reprodutiva I, as ovelhas tiveram um grande número de partos duplos (T1 27\% e T2 42\%), fator que pode ter ocasionado um maior desgaste, principalmente no tratamento 2, e, com isso, uma maior perda de peso das ovelhas durante a lactação. Na estação II, as ovelhas iniciaram a parição no mês de março de 2002, e a desmama ocorreu numa época em que a qualidade da pastagem já estava em declínio devido a proximidade do inverno. As menores médias de peso foram obtidas aos 70 dias de lactação. GODFREY et al. (1997) também observaram perdas de peso, no entanto, as maiores perdas ocorreram entre os 21 e 28 dias de lactação.

As médias de condição corporal estão na tabela 2. A condição corporal inicial (CCI) foi maior $(\mathrm{P}<0,05)$ para o $\mathrm{T} 2=2,47$ e para o $\mathrm{T} 1=2,16$ e na condição corporal final (CCF), 2,19 e 2,60, respectivamente para os T1 e T2, mas não diferiu entre os tratamentos na CC pré-parto. Em relação aos genótipos, a diferença na CCI da mestiça Santa Inês-Suffolk foi 2,40 e a da Santa Inês foi 2,22, sendo significativa $(\mathrm{P}<0,05)$. A CCF da mestiça Santa Inês-Suffolk foi 2,49, sendo também maior que a condição corporal da Santa Inês, que obteve 2,30, mas não diferiram na CC pré-parto. Em relação às três estações reprodutivas, apenas na estação I a CCI= 2,55, CCF= 2,8 e a CCPP= 3,03 foram significativamente maiores $(\mathrm{P}<0,05)$. Já as estações reprodutivas $\mathrm{II}=2,47$ e $\mathrm{III}=2,10$ diferiram somente na CCPP, sendo menor na estação III. Como houve diminuição no intervalo entre partos, as ovelhas na estação III, apesar de terem recuperado o peso corporal, provavelmente não conseguiram recuperar a condição

Tabela 2 - Médias de condição corporal no início(CCI), no final (CCF), no pré-parto (CCPP), ao parto (CCPART), aos 30 dias (CC30d), 70dias (CC70d) e 100 dias de lactação (CC100d) das raças (SI) e (SIxSF) nos tratamentos 1 e 2 das estações reprodutivas I, II e III.

\begin{tabular}{|c|c|c|c|c|c|c|c|}
\hline & $\mathrm{T} 1$ & $\mathrm{~T} 2$ & SI & SF & Est.I & Est.II & Est.III \\
\hline CCI & $2,16^{\mathrm{b}}$ & $2,47^{\mathrm{a}}$ & $2,22^{\mathrm{b}}$ & $2,40^{\mathrm{a}}$ & $2,69^{\mathrm{a}}$ & $2,15^{b}$ & $2,10^{\mathrm{b}}$ \\
\hline CCF & $2,19^{b}$ & $2,60^{\mathrm{a}}$ & $2,50^{\mathrm{b}}$ & $2,49^{a}$ & $2,80^{\mathrm{a}}$ & $2,26^{\mathrm{b}}$ & $2,12^{\mathrm{b}}$ \\
\hline ССРР & 2,40 & 2,66 & 2,42 & 2,65 & $3,03^{\mathrm{a}}$ & $2,47^{\mathrm{b}}$ & $2,10^{\mathrm{b}}$ \\
\hline CCPART & 2,06 & 2,16 & 2,12 & 2,10 & $2,55^{\mathrm{a}}$ & $1,99^{\mathrm{b}}$ & $1,78^{b}$ \\
\hline CC30d & $1,87^{\mathrm{b}}$ & $2,06^{a}$ & $2,04^{\mathrm{a}}$ & $1,89^{b}$ & $2,20^{\mathrm{a}}$ & $2,11^{\mathrm{a}}$ & $1,59^{b}$ \\
\hline CC70d & $1,57^{\mathrm{b}}$ & $1,87^{\mathrm{a}}$ & 1,75 & 1,69 & $1,90^{\mathrm{a}}$ & $1,83^{\mathrm{a}}$ & $1,43^{b}$ \\
\hline CC100d & $1,58^{\mathrm{b}}$ & $1,91^{\mathrm{a}}$ & $1,81^{\mathrm{a}}$ & $1,69^{\mathrm{b}}$ & $2,01^{\mathrm{a}}$ & $1,63^{\mathrm{b}}$ & $1,60^{\mathrm{b}}$ \\
\hline
\end{tabular}


corporal no período pré-parto. GONZALEZ et al. (1997) avaliaram no Uruguai, durante três anos seguidos, em rebanhos de diferentes raças, o efeito do peso da ovelha e sua condição corporal no período de encarneiramento, quanto à eficiência reprodutiva, e encontraram uma condição corporal média das ovelhas que não pariram, pariram um ou dois cordeiros de 2,68; 3,08 e 3,13, respectivamente.

A condição corporal no parto não variou entre os tratamentos e nem entre as raças, mas entre as estações houve diferença significativa $(\mathrm{P}<0,05)$, sendo que, na estação reprodutiva I, a condição corporal no parto foi 2,55, sendo maior, comparada com as estações reprodutivas $\mathrm{II}=(1,99)$ e III= $(1,78)$, e estas não foram diferentes entre si. Já aos 30 dias de lactação, ocorreram diferenças $(\mathrm{P}<0,05)$ entre tratamentos ( $\mathrm{T} 1$ 1,87 e T2 2,06), entre raças (Santa Inês= 2,04 e mestiças Santa InêsSuffolk=1,89) e entre as estações reprodutivas (Est. I= 2,20 e II=2,11), as quais não diferiram entre si; porém, quando comparadas com a estação III $=(1,58)$, houve diferença $(\mathrm{P}<0,05)$. Aos 70 dias de lactação, ocorreu diferença entre os tratamentos 1 e 2 (1,57 e 1,87, respectivamente).

Quanto ao efeito de época, não houve diferença entre as estações reprodutivas I e II (1,90 e 1,83, respectivamente), as quais diferiram da estação III $(1,43)$. Isto possivelmente aconteceu porque, na estação reprodutiva III, as ovelhas já estavam mais desgastadas devido ao maior número de crias. Quanto aos 100 dias pós-parto houve, efeito de tratamento (T1= 1,58 e T2=1,91), de raça (SI=1,81 e SIxSF=1,69) e de estação reprodutiva (I= 2,01, II=1,63 e III=1,60).

De acordo com os resultados obtidos, verificou-se efeito de estação de monta sobre a fertilidade em ambos os genótipos e no tratamento 1 , que não foi suplementado com concentrado, e no tratamento 2, suplementado três semanas antes e quatro após o início do manejo para concepção, três semanas antes do parto e durante a lactação. As ovelhas apresentaram, de acordo com a tabela 3, maior fertilidade na primeira estação reprodutiva, nos meses de fevereiro a agosto de 2002. Provavelmente o efeito do concentrado nessa primeira estação reprodutiva teve um maior resultado, pois as ovelhas estavam descansadas, devido a anteriormente estarem em manejo reprodutivo, com um intervalo entre partos mais extenso. FORGATY \& GILMOUR (1998) e RASTOGI (2001) estudaram o efeito da estação de monta sobre o desempenho reprodutivo de rebanho selecionado para não ter estacionalidade reprodutiva durante todo o ano ou não, testados em sistemas de parição a cada oito meses, e encontraram efeito da estação na fertilidade e sobrevivência de cordeiros. Na estação reprodutiva I e
Tabela 3 - Porcentagens de fertilidade das raças SI e SF e de partos duplos, nos T1 e T2, durante as estações reprodutivas I, II e III.

\begin{tabular}{lllll}
\hline Tratamento & Raça & Est.I & Est.II & Est.III \\
\hline T1 (\% fertilidade) & SI & 70,0 & 38,0 & 54,0 \\
& SIxSF & 75,0 & 42,0 & 60,0 \\
T2(\% fertilidade) & SI & 88,0 & 50,0 & 76,0 \\
T1 & SIxSF & 77,0 & 56,0 & 87,0 \\
(\% partos duplos) & & 30,0 & 4,0 & 8,7 \\
T2 & & & & \\
(\% partos duplos) & & 47,0 & 6,0 & 25,0 \\
\hline
\end{tabular}

T1 (não suplementado), ambas as raças tiveram 72,5\% de fertilidade. No T2 (suplementado), a mestiça Santa Inês-Suffolk apresentou $77 \%$ e a Santa Inês $88 \%$ de fertilidade. Entretanto, na estação II, a fertilidade foi de $44 \%$ para as ovelhas mestiças e de $38 \%$ para as ovelhas Santa Inês, e o T2 resultou em 56\% e 50\% de fertilidade para a mestiça Santa Inês-Suffolk e para a Santa Inês, respectivamente. Na estação III, a fertilidade no T1 foi de $60 \%$ para a mestiça Santa Inês-Suffolk e de 54\% para a Santa Inês, e, no T2, de $87 \%$ e $76 \%$ respectivamente para a mestiça Santa Inês-Suffolk e para a Santa Inês. RODA et al. (1993) acasalaram ovelhas da raça Ideal, Corriedale e Suffolk a cada oito meses e observaram 33,1; 24,1 e 42,8\% de acasalamentos férteis nos meses de janeiro, setembro e maio, respectivamente, possibilitando, com isso, três parições a cada dois anos. Também NOTTER \& COPEHAVER (1980), comparando o desempenho de 183 ovelhas acasaladas em diferentes meses, durante um período de 5 anos, com desmame aproximadamente aos 43 dias de idade, também constataram variações nas taxas de concepção em agosto (90\%), novembro (79\%) e abril (53\%). Já ANDRIGUETTO et al. (1998) não encontraram efeito da restrição alimentar na fertilidade de ovelhas acasaladas em época desfavorável.

Verificou-se diferença significativa entre os tratamentos na prolificidade das ovelhas. Na tabela 3, observa-se 1,20 e 1,55 na estação reprodutiva I (fevereiro a agosto de 2002), na Est.II (outubro de 2002 a maio de 2003), de 0,90 e 1,03 e, na Est.III (julho de 2003 à fevereiro de 2004), de 1,11 e 1,14, respectivamente para os tratamentos 1 e 2 . Não ocorreu diferença entre os genótipos. Resultados semelhantes foram observados por RIBEIRO et al. (2001) no norte do Paraná, com maior taxa de natalidade para ovelhas acasaladas no outono (80\%), quando comparado com o acasalamento no verão (60\%). BIANCHI et al. (2001) estudaram o manejo pós-desmame de ovelhas da raça Merino Australiano 
no Uruguai, e observaram que as taxas de ovulação para ovelhas com CC média no início da monta de 2,$40 ; 2,70$ e 3,40 foram de 1,20; 1,18 e 1,32, respectivamente.

\section{CONCLUSÕES}

O sistema de manejo em que as ovelhas são suplementadas três semanas antes e quatro após o início do manejo para concepção, três semanas antes do parto e durante a lactação influencia positivamente o peso, a condição corporal, a fertilidade e a prolificidade das ovelhas.

\section{REFERÊNCIAS}

BIANCHI, G. et al. Manejo alimentar pós-desmame e performance de ovelhas Merino em pastagens naturais e artificiais na época de encarneiramento. Ciência Rural, v.31, n.1, p.105-110, 2001.

DUCKER, M.J.; BOYD, J. The effect of body size and body conditional on the ovulation rate of ewes. Animal Production, v.24, p.377-385, 1977.

FORGATY, N.M.; GILMOUR, A.R. Effect of season on lambing performance of selection and control flock ewes tested in a 8-monthly lambing system. Wool Technology and sheep breeding, v.46, p.286-290, 1998.

GODFREY, R.W. et al. Lamb growth and milk production of hair and wool sheep in a semi-arid tropical environment. Small Ruminant Research, v.24, p.77-83, 1997.

GONZALES, R.E. et al. The effects of ewe live weigtht and body conditional score around mating on production from four sheep breeds in extensive grazing systems in Uruguay. Animal Science, v.64, p.139-145, 1997.

GORDON, H.M; WHITLOCK, H.V. A new technique four counting nematode eggs in sheep faeces. Journal Counc Science Ind Research, v.12, p.50-52, 1939.
GUNN, R.G., et al. Effects of age and its relationship with body size and reproductive performance in scottish blackface ewes. Animal Production, v.43, p.279-283, 1984.

GUNN, R.G., et al. The effect of level of nutritional prior to mating on the reproductive performance of ewes of two welsh breeds in different levels of body conditional. Animal Production, v.52, p.157-163, 1991.

MORLEY, F.H.W. et al. Predicting ovulation rate from live weigth in ewes. Animal Sciense, v.3, p.27-45, 1978.

NATIONAL RESARCH COUNCIL - NRC. Nutrient requirements of sheep. 6.ed. Washington, DC: National Academy of Sciences, 1985. 99p.

NOTTER, D.R; COPENHAYER, J.S. Performance of finnish landrace crossbred ewes under accelerated lambing. I. Fertility, prolificacy and ewe productivity. Animal Science, v.51, n.5, p.1033-1042, 1980.

RASTOGI, R.K. Production performance of Barbados blackbelly sheep in Tobago, West Indies. Small Ruminant Research, v.41, p.171-175, 2001.

RIBEIRO, E.L.A. et al. Desempenho reprodutivo de ovelhas encarneiradas no verão ou no outono na região norte do Paraná. In: REUNIÃO DA SOCIEDADE BRASILEIRA DE ZOOTECNIA, 2001, Piracicaba. Anais... Piracicaba: SBZ, 2001. p.497-502.

RIBEIRO, L.A.O. Relação entre a condição corporal e a idade das ovelhas no encarneiramento com a prenhez. Ciência Rural, Santa Maria, v.33, n.2, p.357-361, 2003.

RODA, D.S. et al. Desempenho de ovinos em sistema de acasalamento a cada 8 meses. Boletim da Indústria Animal, v.50, n.1, p.49-54, 1993.

SAEG. (UNIVERSIDADE FEDERAL DE VIÇOSA) UFV. Sistema de Análises Estatísticas e Genéticas. Versão 7.0 Viçosa-MG, 2000. 150p. (Manual do usuário).

SUSIN, I. Exigências nutricionais de ovinos e estratégias de alimentação. In: SILVA SOBRINHO, et al. Nutrição de ovinos. Jaboticabal: FUNEP, 1996. p.119-141. 